\title{
Thermal-mechanical deformation modelling of soft tissues for thermal ablation
}

\author{
Xin Li, Yongmin Zhong ${ }^{*}$, Reza Jazar and Aleksandar Subic \\ School of Aerospace, Mechanical and Manufacturing Engineering, RMIT University, Bundoora, VIC \\ 3083, Australia
}

\begin{abstract}
Modeling of thermal-induced mechanical behaviors of soft tissues is of great importance for thermal ablation. This paper presents a method by integrating the heating process with thermal-induced mechanical deformations of soft tissues for simulation and analysis of the thermal ablation process. This method combines bio-heat transfer theories, constitutive elastic material law under thermal loads as well as non-rigid motion dynamics to predict and analyze thermal-mechanical deformations of soft tissues. The 3D governing equations of thermal-mechanical soft tissue deformation are discretized by using the finite difference scheme and are subsequently solved by numerical algorithms. Experimental results show that the proposed method can effectively predict the thermal-induced mechanical behaviors of soft tissues, and can be used for the thermal ablation therapy to effectively control the delivered heat energy for cancer treatment.
\end{abstract}

Keywords: Thermal ablation, soft tissue, bio-heat transfer, thermo-mechanical deformation

\section{Introduction}

Thermal ablation is a minimally invasive therapy for patients with cancer, particularly applicable for patients with unrespectable hepatic tumors. The procedure uses energy sources such as radiofrequency [1], laser [2], focused ultrasound and microwaves [3,4] to generate thermal injury to cells within the tumor. On implementing this treatment, current surgical procedure always comes with problems such as 1) inadequate imaging modalities; 2) non-optimized power supply to form an adequate ablation area; and 3) the inability to form an ablation area of sufficient size for large tumors. These shortcomings make it difficult to control the precise formation of coagulation zones, that is, it is difficult to affect a volume sufficient to cover the tumor not surrounding safety area. Additionally, complications such as burns to neighboring organs or the bile duct often occur due to excessive heat transfer. These problems are crucial for the patients because tumors often recur from areas that have not been fully cauterized. To overcome these difficulties, considerable research efforts have been dedicated to modeling and analysis of this treatment process. However, most of the existing methods only focused on temperature indication in order to predict the size of the volume affected by delivered heat, while neglecting or underestimating the thermal-mechanical deformation during this procedure. In fact, together with rising temperature within heated tissue, even a relatively small variation in

\footnotetext{
${ }^{*}$ Corresponding author: Yongmin Zhong, School of Aerospace, Mechanical and Manufacturing Engineering, RMIT University, Bundoora, VIC 3083, Australia. Tel.: +61399256018. Fax: +61399256018. E-mail: yongmin.zhong@rmit.edu.au.
} 
thermal induced mechanical deformation can lead to various effects such as altered production of hormones, suppressed immune response, and protein denaturation [5].

This paper presents a method for modeling of thermal-mechanical soft tissue deformation for thermal ablation. This method integrates both the bio-heat process and thermal-induced mechanical deformation process to mimic and analyze the procedure of thermal ablation. A thermal-mechanical model of soft tissues is established by combing bio-heat transfer theories, constitutive elastic material law under thermal loads and non-rigid motion dynamics. The finite difference scheme and iterative Gauss-Seidel algorithms are utilized in the construction of the model. Simulations have been conducted to verify the efficiency of the proposed method for thermal ablation.

\section{Related work}

Significant research efforts are dedicated to modeling and analysis of thermally induced soft tissue behaviors. Various mathematical and computer-based simulation models have been proposed according to different types of heating sources. Prakash et al. established a 3D acoustic bio-thermal model $[3,6]$ based on COMSOL, in which both acoustic energy distributions and transient temperature profiles in tissue during heating were investigated. However, the determination of thermal dose for the heating source of ultrasound still relies on temperature indication. For radio frequency heating sources, Watanabe et al used the Finite Element Method to simulate temperature distribution in the target organ and subsequently generate the size and shape of coagulation zone based on the dimensions of the tumor $[7,8]$. In terms of microwave ablation, Rattanadecho et al. reported an axisymmetric Finite Element Method to study heat transfer and blood flow, coupled with electromagnetic wave propagation in liver tissue [9]. Generally, all the above models only focus on the indication of thermally induced temperature distribution (or related conclusions) to generate coagulation zone and determine a thermal dose, while neglecting or underestimating the accompanied phenomenon of thermal-mechanical deformation of target tissue and its effect on making the above decisions.

Research efforts have also been dedicated to studying thermal mechanical properties of human skin for skin burn. Under the common assumption that human skin is uniformly linear and orthotropic within a certain area, various numerical models were developed to simulate skin burn process. Xu et al. investigated a mathematical approach to examine the heat transfer process, thermal damage and the heat-induced mechanical response of human skin [10,11]. Shen et al. reported a quasi-steady state numerical model, to predict the thermo-elastic deformation of skin tissue at high temperature $[12,13]$. Lozano et al. analyzed the RF heating of cutaneous and subcutaneous tissues, including their thermoelastic response based on the commercialized analysis software COMSOL [14]. Among these models, $\mathrm{Xu}$ et al. and Shen et al. employed a similar concept, upon using quasi-steady state thermal mechanical models, which assumed that the thermal mechanical deformation induced by temperature difference at a selected point was a steady state calculation result within two successive time points. The difference between their models and that proposed by Lozano et al., lies in the motion equation employed, where Lozano et al considered that the thermal mechanical deformation process was dynamic in time domain, which is more realistic, except for the entire simulation was conducted based on a commercially available Finite Element Method tool.

The discretization methods applied to solve related mathematical models established in this field can be classified into two categories: Finite Difference Method (FDM) [12,13] and Finite Element Method (FEM) [3,6-9,14-17]. Considering the high computational cost of FEM in dealing with largescale 3D problems, the FDM method was selected in this article for doing our integrated simulation, 
because of its maturity and simplicity. Based on a coupled bio-heat transfer theories, constitutive elastic material law under thermal loads and non-rigid motion dynamics, the established thermal ablation simulator in this article is able to simulate both heating process and thermal-mechanical deformation within target tissue. From the viewpoint of surgery planning, the simulation results can assist surgeons to view a more realistic picture of the temperature change within the heated tissue, as well as its deformed size, based on which method of delivered heat can be optimized before a real surgery is performed.

\section{Proposed thermal-mechanical model}

In general, by ignoring the thermo-elastic coupling during this procedure, the calculation of thermalmechanical deformation of soft tissues under a particular form of thermal load can be treated as two decoupled parts: a temperature distribution calculation and a rising temperature induced mechanical deformation calculation. Firstly, temperature field is obtained from bio-heat transfer equations. The temperature distribution is used as an input, when the displacement field is calculated by applying constitutive elastic material law under thermal loads and non-rigid motion dynamics.

\subsection{Bio-heat transfer}

Bio-heat transfer conduction with a continuous internal heat source within a soft tissue, is described by a partial differential equation of the second order,

$$
\rho C \frac{\partial T}{\partial t}=k\left(\frac{\partial^{2} T}{\partial x^{2}}+\frac{\partial^{2} T}{\partial y^{2}}+\frac{\partial^{2} T}{\partial z^{2}}\right)+Q
$$

where $\rho$ is the tissue density, $\mathrm{C}$ the tissue specific heat, $\mathrm{k}$ the tissue thermal conductivity, $\mathrm{T}$ the temperature at time $t, \mathrm{Q}$ the heat source, here, $\mathrm{Q}$ defines the combined effect of externally applied heating and internal cooling, such as blood perfusion.

To solve Eq. (1), it is necessary to determine the boundary conditions. The boundary conditions determine the character of the heat interchange over the boundary. For simplicity, the Dirichlet boundary conditions was chosen as shown in (2), i.e. the specified temperature on the boundary $\Omega$ :

$$
T=T_{\Omega} \text { on } \Omega
$$

\subsection{Constitutive elastic material law under thermal loads}

If the human tissue is treated as a kind of elastic material $[10,18]$, under a thermal load, the constitutive strain-stress equations for a homogenous, isotropic human tissue in three-dimensional Cartesian coordinates $(\mathrm{x}, \mathrm{y}, \mathrm{z})$ can be described as

$$
\begin{aligned}
& \varepsilon_{x x}=\frac{1}{E}\left[\sigma_{x x}-v\left(\sigma_{y y}+\sigma_{z z}\right)\right]+\alpha \tau, \quad \varepsilon_{y y}=\frac{1}{E}\left[\sigma_{y y}-v\left(\sigma_{x x}+\sigma_{z z}\right)\right]+\alpha \tau \\
& \varepsilon_{z z}=\frac{1}{E}\left[\sigma_{z z}-v\left(\sigma_{y y}+\sigma_{x x}\right)\right]+\alpha \tau, \varepsilon_{x y}=\frac{1+v}{E} \sigma_{x y}, \quad \varepsilon_{x z}=\frac{1+v}{E} \sigma_{x z}, \quad \varepsilon_{y z}=\frac{1+v}{E} \sigma_{y z}
\end{aligned}
$$


where $\varepsilon$ is the normal strain, $\sigma$ the normal stress, $\tau$ the temperature difference at two successive time points, E the Young's modulus, $v$ the Poisson's ratio, and $\alpha$ the linear coefficient of thermal expansion. Eq. (3) can be further rewritten as:

$$
\begin{aligned}
& \sigma_{x x}=\frac{E}{1+v} \varepsilon_{x x}+\frac{v E}{(1+v)(1-2 v)}\left(\varepsilon_{x x}+\varepsilon_{y y}+\varepsilon_{z z}\right)-\frac{\alpha E}{(1-v)} \tau \\
& \sigma_{y y}=\frac{E}{1+v} \varepsilon_{y y}+\frac{v E}{(1+v)(1-2 v)}\left(\varepsilon_{x x}+\varepsilon_{y y}+\varepsilon_{z z}\right)-\frac{\alpha E}{(1-v)} \tau \\
& \sigma_{z z}=\frac{E}{1+v} \varepsilon_{z z}+\frac{v E}{(1+v)(1-2 v)}\left(\varepsilon_{x x}+\varepsilon_{y y}+\varepsilon_{z z}\right)-\frac{\alpha E}{(1-v)} \tau \\
& \sigma_{x y}=\frac{E}{1+v} \varepsilon_{x y}, \quad \sigma_{x z}=\frac{E}{1+v} \varepsilon_{x z}, \sigma_{y z}=\frac{E}{1+v} \varepsilon_{y z}
\end{aligned}
$$

The strain $\varepsilon$ can be further described by the displacement vector $\left(\mu_{\mathrm{x}}, \mu_{\mathrm{y}}, \mu_{\mathrm{z}}\right)$ as

$$
\begin{aligned}
& \varepsilon_{x x}=\frac{\partial \mu_{x}}{\partial x}, \quad \varepsilon_{y y}=\frac{\partial \mu_{y}}{\partial y}, \quad \varepsilon_{z z}=\frac{\partial \mu_{z}}{\partial z} \\
& \varepsilon_{x y}=\frac{1}{2}\left(\frac{\partial \mu_{x}}{\partial y}+\frac{\partial \mu_{y}}{\partial x}\right), \quad \varepsilon_{x z}=\frac{1}{2}\left(\frac{\partial \mu_{x}}{\partial z}+\frac{\partial \mu_{z}}{\partial x}\right), \quad \varepsilon_{y z}=\frac{1}{2}\left(\frac{\partial \mu_{y}}{\partial z}+\frac{\partial \mu_{z}}{\partial y}\right)
\end{aligned}
$$

\subsection{Non-rigid motion dynamics}

The govern equation for the non-rigid mechanics of motion can be written as

$$
\begin{aligned}
& \rho \frac{\partial^{2} \mu_{x}}{\partial t^{2}}=\frac{\partial \sigma_{x x}}{\partial x}+\frac{\partial \sigma_{x y}}{\partial y}+\frac{\partial \sigma_{x z}}{\partial z}+F_{x}, \rho \frac{\partial^{2} \mu_{y}}{\partial t^{2}}=\frac{\partial \sigma_{y x}}{\partial x}+\frac{\partial \sigma_{y y}}{\partial y}+\frac{\partial \sigma_{y z}}{\partial z}+F_{y} \\
& \rho \frac{\partial^{2} \mu_{z}}{\partial t^{2}}=\frac{\partial \sigma_{z x}}{\partial x}+\frac{\partial \sigma_{z y}}{\partial y}+\frac{\partial \sigma_{z z}}{\partial z}+F_{z}
\end{aligned}
$$

The generated temperature distribution field with respect to time, obtained from Eq. (1) is used as the input of the thermo-elasticity model Eq. (3) to obtain the corresponding thermal stress. Subsequently, the thermo-elastic displacement can be determined according to non-rigid mechanics of motion, i.e. Eq. (6). Thus, by combining all the above governing Eqs. (1)-(6), the displacement due to a thermal load can be described as

$$
\rho \frac{\partial^{2} \mu_{x}}{\partial t^{2}}=\frac{E}{2(1+v)}\left(\frac{\partial^{2} \mu_{x}}{\partial x^{2}}+\frac{\partial^{2} \mu_{x}}{\partial y^{2}}+\frac{\partial^{2} \mu_{x}}{\partial z^{2}}\right)+\frac{E}{2(1+v)(1-2 v)} \frac{\partial}{\partial x}\left(\frac{\partial \mu_{x}}{\partial x}+\frac{\partial \mu_{y}}{\partial y}+\frac{\partial \mu_{z}}{\partial z}\right)-\frac{E \alpha}{1-2 v} \frac{\partial \tau}{\partial x}+F_{x}
$$




$$
\begin{aligned}
& \rho \frac{\partial^{2} \mu_{y}}{\partial t^{2}}=\frac{E}{2(1+v)}\left(\frac{\partial^{2} \mu_{y}}{\partial x^{2}}+\frac{\partial^{2} \mu_{y}}{\partial y^{2}}+\frac{\partial^{2} \mu_{y}}{\partial z^{2}}\right)+\frac{E}{2(1+v)(1-2 v)} \frac{\partial}{\partial y}\left(\frac{\partial \mu_{x}}{\partial x}+\frac{\partial \mu_{y}}{\partial y}+\frac{\partial \mu_{z}}{\partial z}\right)-\frac{E \alpha}{1-2 v} \frac{\partial \tau}{\partial y}+F_{y} \\
& \rho \frac{\partial^{2} \mu_{z}}{\partial t^{2}}=\frac{E}{2(1+v)}\left(\frac{\partial^{2} \mu_{z}}{\partial x^{2}}+\frac{\partial^{2} \mu_{z}}{\partial y^{2}}+\frac{\partial^{2} \mu_{z}}{\partial z^{2}}\right)+\frac{E}{2(1+v)(1-2 v)} \frac{\partial}{\partial z}\left(\frac{\partial \mu_{x}}{\partial x}+\frac{\partial \mu_{y}}{\partial y}+\frac{\partial \mu_{z}}{\partial z}\right)-\frac{E \alpha}{1-2 v} \frac{\partial \tau}{\partial z}+F_{z}
\end{aligned}
$$

\section{Model construction}

The proposed model can be constructed on both regular and irregular volume meshes. For simplicity, model constructed on regular volume meshes is considered in this paper.

The construction of the proposed model on a regular volume can be established by using a finite difference scheme. For a cubic volume mesh as illustrated in Figure 1(a), a fully implicit difference method based on the central difference approximation, is adopted for solving the bio-heat conduction equation, Eq. (1). For example, the discretized bio-heat conduction equation at point $(\mathrm{i}, \mathrm{j}, \mathrm{k})$ can be described by its seven neighboring points (see Figure 1(a)) as:

$$
\begin{aligned}
& \frac{\partial T}{\partial t}_{t+1}=\frac{T_{i, j, k, t+1}-T_{i, j, k, t}}{\Delta t}, \quad \frac{\partial^{2} T}{\partial x^{2} t+1}=\frac{T_{i+1, j, k, t+1}+T_{i-1, j, k, t+1}-2 T_{i, j, k, t+1}}{\Delta x_{i}{ }^{2}} \\
& \frac{\partial^{2} T}{\partial y^{2}}{ }_{t+1}=\frac{T_{i, j+1, k, t+1}+T_{i, j-1, k, t+1}-2 T_{i, j, k, t+1}}{\Delta y_{i}{ }^{2}} \\
& \frac{\partial^{2} T}{\partial z^{2}}{ }_{t+1}=\frac{T_{i, j, k+1, t+1}+T_{i, j, k-1, t+1}-2 T_{i, j, k, t+1}}{\Delta z_{i}{ }^{2}} \\
& \frac{\rho C}{k} \cdot \frac{T_{i, j, k, t+1}-T_{i, j, k, t}}{\Delta t}-\frac{Q}{k}=\frac{T_{i+1, j, k, t+1}+T_{i-1, j, k, t+1}-2 T_{i, j, k, t+1}}{\Delta x_{i}{ }^{2}}+\frac{T_{i, j+1, k, t+1}+T_{i, j-1, k, t+1}-2 T_{i, j, k, t+1}}{\Delta y_{i}{ }^{2}}+ \\
& \frac{T_{i, j, k+1, t+1}+T_{i, j, k-1, t+1}-2 T_{i, j, k, t+1}}{\Delta z_{i}^{2}}
\end{aligned}
$$

Similarly, the discretized thermal-mechanical deformation equation at point (i,j,k) can be described using its neighboring points (see Figure 1(a)). For, example, the discretization process for the first equilibrium of Eq. (7) that covers the displacement calculation of all inner points in the $\mathrm{x}$-axis direction is described as follows:

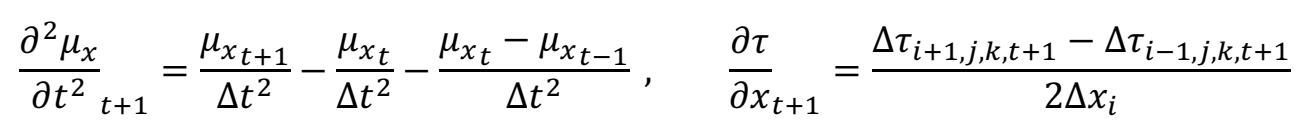

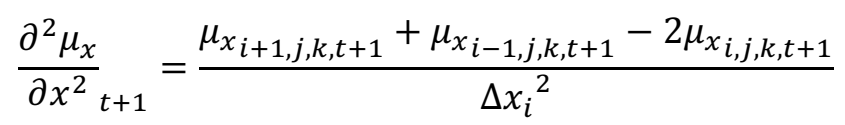

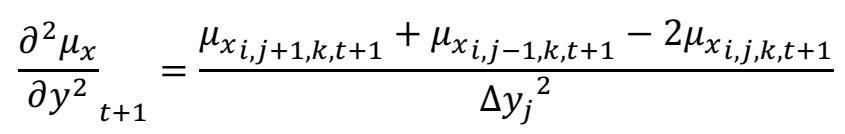




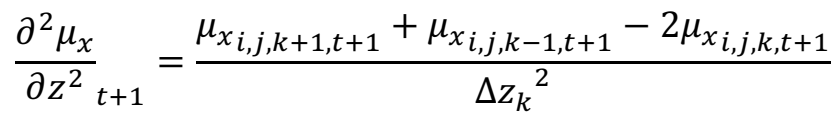

$$
\begin{aligned}
& \frac{\partial}{\partial x}\left(\frac{\partial \mu_{y}}{\partial y}\right)_{t+1}=\frac{\mu_{y_{i+1, j+1, k, t+1}}-\mu_{y_{i+1, j-1, k, t+1}}-\mu_{y_{i-1, j+1, k, t+1}}+\mu_{y_{i-1, j-1, k, t+1}}}{4 \Delta x_{i} \Delta y_{j}} \\
& \frac{\partial}{\partial x}\left(\frac{\partial \mu_{z}}{\partial z}\right)_{t+1}=\frac{\mu_{z i+1, j, k+1, t+1}-\mu_{z i+1, j, k-1, t+1}-\mu_{z i-1, j, k+1, t+1}+\mu_{z i-1, j, k-1, t+1}}{4 \Delta x_{i} \Delta z_{k}}
\end{aligned}
$$

Substituting the various discretized components of displacement stated as above into Eq. (6), displacement of all inner points under thermal loads could be expressed accordingly.

Solving the above-discretized equations into computer in time domain, an iterative Gauss-Seidel method is employed, because of its faster convergence rate and lower storage requirement.

\section{Implementation and results}

A prototype system has been implemented for heating and thermo-mechanical interaction within the target tissue. This system allows users to change both material properties and the applied heating power. Experiments were conducted to investigate the performance of the proposed the model.

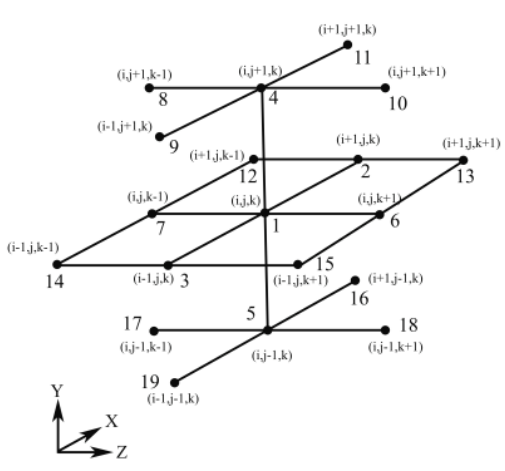

(a)

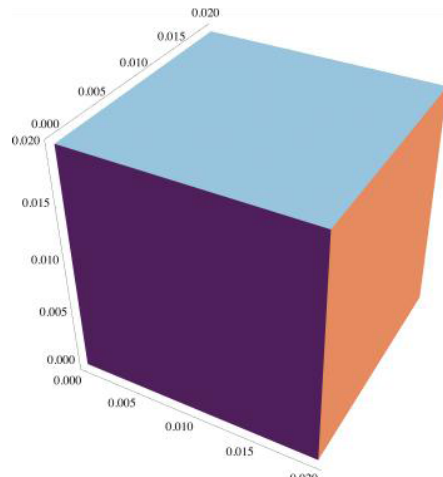

(b)

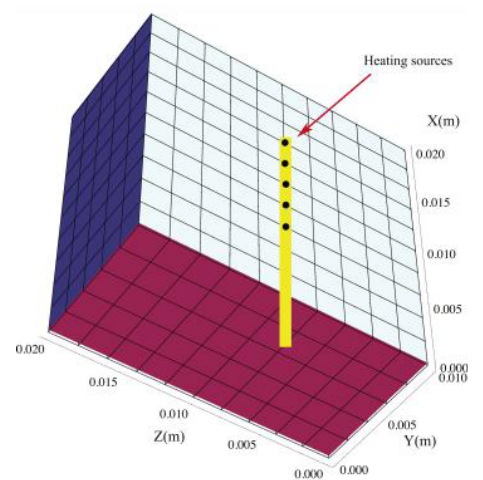

(c)

Fig. 1. A cubic grid (a), the dimensions of a tissue model for simulation (b) and the Y-axis half section view of five points heating sources inside the tissue model (c).

Table 1

Tissue parameters and constants

\begin{tabular}{lc}
\hline Parameters & Value \\
\hline Thermal conductivity k; W/mK & 0.613 \\
Specific heat $\mathrm{C} ; \mathrm{j} / \mathrm{kgK}$ & 4192 \\
Thermal expansion coefficient $\alpha ; 1 /{ }^{\circ} \mathrm{C}$ & $1 \times 10^{-4}$ \\
Density $\rho ; \mathrm{kg} / \mathrm{m}^{3}$ & 1060 \\
Young's modulus $\mathrm{E} ; \mathrm{Pa}$ & $10.2 \times 10^{6}$ \\
Shear modulus $\mathrm{G} ; \mathrm{Pa}$ & $3.7 \times 10^{6}$ \\
Poisson's ratio $v ;$ & 0.35 \\
\hline
\end{tabular}


Trials were conducted to evaluate the performance of the proposed model in terms of a cubic-shape tumor model in the size of $20 \mathrm{~mm} \times 20 \mathrm{~mm} \times 20 \mathrm{~mm}$, as shown in Figure 1(b). The tumor model was composed of 1000 cubic elements with 1331 nodes. The needle used in thermal ablation was modeled as a line, with five evenly spaced points on the mesh as heat sources (the first one is located at the tip of the needle), for generating heat energy. As shown in Figure 1(c), the needle was positioned such that the middle of five heating points was located at the center of the cubic volume tumor model. It generated thermal energy through the five points at $1.0 \times 10^{7} \mathrm{~W} / \mathrm{m}^{3}$ to heat up the tumor model. Since we only consider mechanical deformation induced by thermal energy in our implementation, external force attached on the tumor model were set to zero. The thermal and mechanical properties of the simulated tumor are listed in Table 1. [17,18].

\subsection{Temperature distribution}

Figure 2 shows the temperature changes at the five heating source points where thermal energy was directly applied in the first 500 seconds. It can be seen that the increase rate of temperature was different before $330 \mathrm{~K}$ and after $330 \mathrm{~K}$. Temperature was increasing rapidly before $330 \mathrm{~K}$, while afterwards, no significant change was observed until the end of simulation time, although heating was continuously applied within this period. In fact, it is this increase rate of temperature that makes the fundamental reason for thermal mechanical deformation, which means theoretically, larger deformation should be observed at points with larger accumulated temperature increase.

For the spatial temperature distribution, Figure 3 shows that the final temperatures of the selected points with respective distances of $0 \mathrm{~m}, 0.012 \mathrm{~m}, 0.014 \mathrm{~m}, 0.016 \mathrm{~m}$ and $0.018 \mathrm{~m}$ from the center of heating source, were descending from $351 \mathrm{~K}$ to $310 \mathrm{~K}$ after 500 seconds of thermal interaction.

Combining Figures 2 and 3, it shows how heat energy was distributed within heated tissue-the temperature at the heating points is higher when compared with surrounding points, while the changing rate reduces with respect to duration of applied heating.

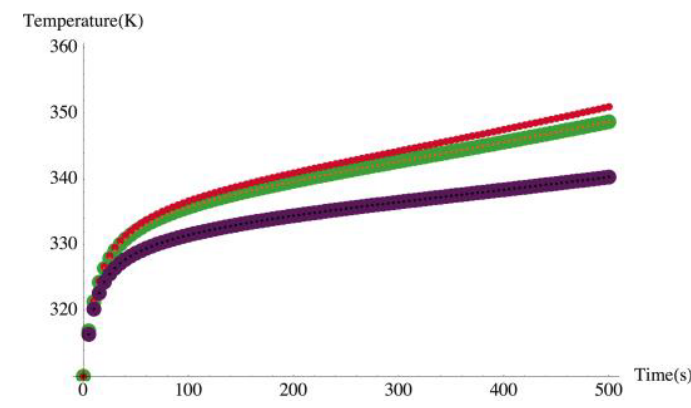

Fig. 2. Temperature at the heating source points.

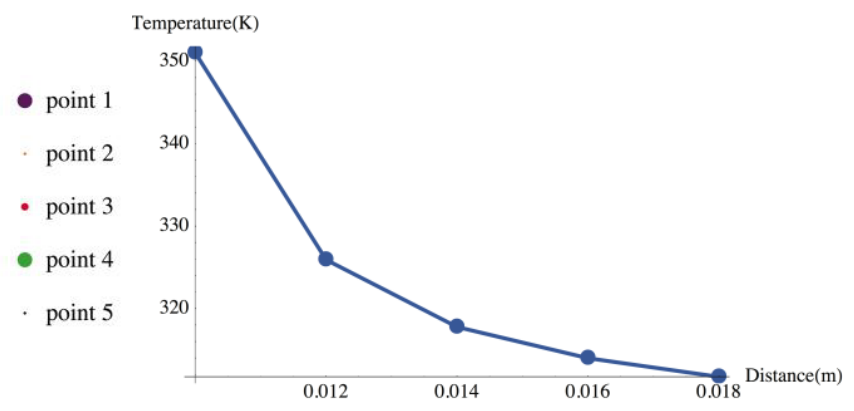

Fig. 3. Temperature change on line $(0.01,0.01,0.01-0.018)$ at $500 \mathrm{~s}$.

\subsection{Displacement distribution}

Figure 4(a) gives the displacement of plane $\mathrm{x}=0.018 \mathrm{~m}$ at the end simulation time of 500 seconds, while Figure 4(b) shows the displacement of its symmetric plane $\mathrm{x}=0.002 \mathrm{~m}$ at the same time point. Considering the position and distribution of applied heating sources, every two selected symmetric planes with regard to the center of heating area were deformed outward as expected, as shown in 
Figures 5(a), 5(b), 6(a), and 6(b). The distance from these planes to the center of heating area was selected to be the same: $0.008 \mathrm{~m}$.

(a)

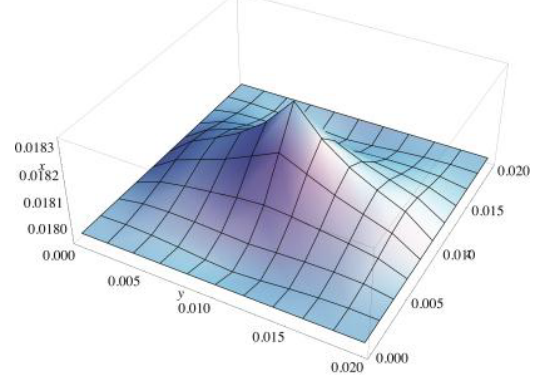

(b)

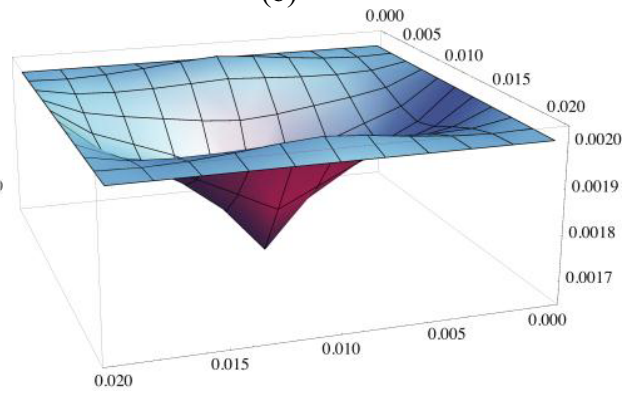

Fig. 4. $X$-axis displacement at plane $X=0.018 \mathrm{~m}$ and $X=0.002 \mathrm{~m}$ at $500 \mathrm{~s}$.

(a)

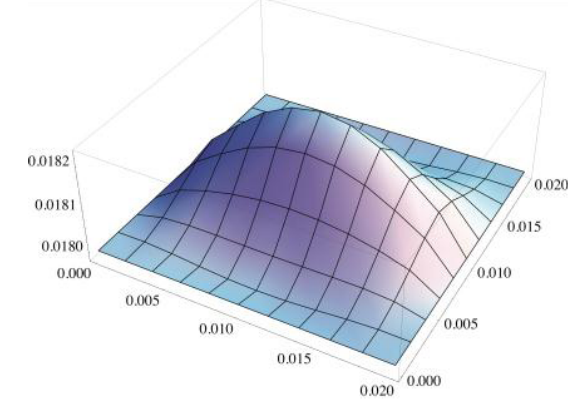

(b)

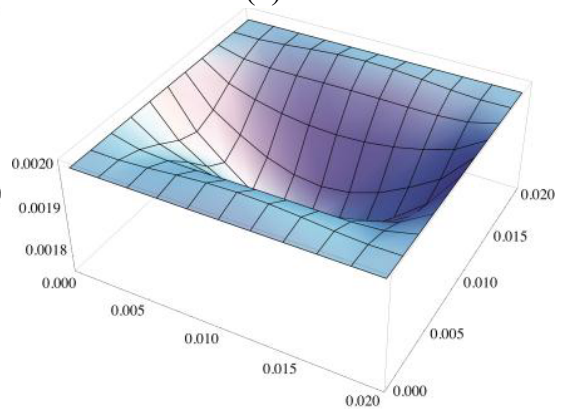

Fig. 5. $\mathrm{Y}$-axis displacement at plane $\mathrm{Y}=0.018 \mathrm{~m}$ and $\mathrm{Y}=0.002 \mathrm{~m}$ at $500 \mathrm{~s}$.

(a)

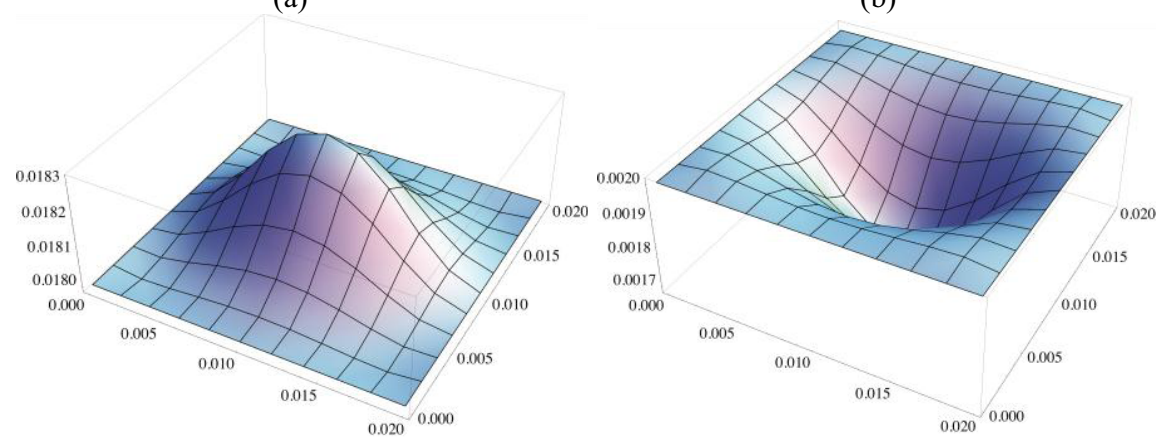

Fig. 6. $\mathrm{Z}$-axis displacement at plane $\mathrm{Z}=0.018 \mathrm{~m}$ and $\mathrm{Z}=0.002 \mathrm{~m}$ at 500 . 


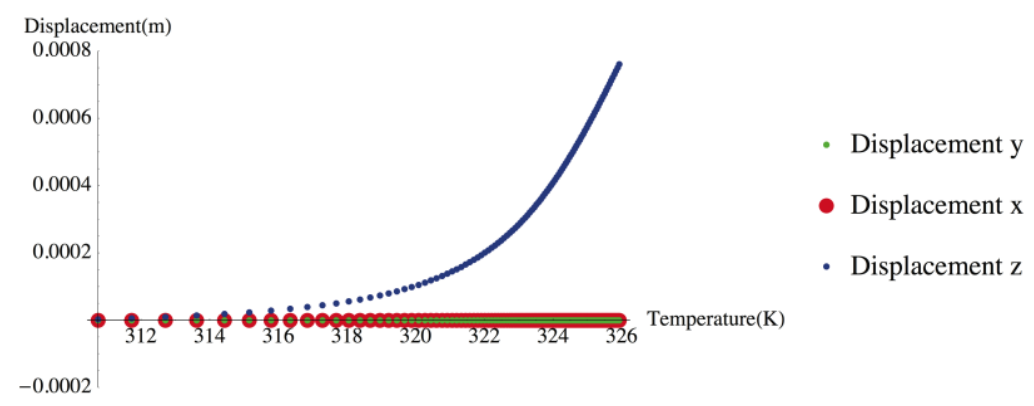

Fig. 7. Displacement changing at point $(0.01,0.01,0.012)$.

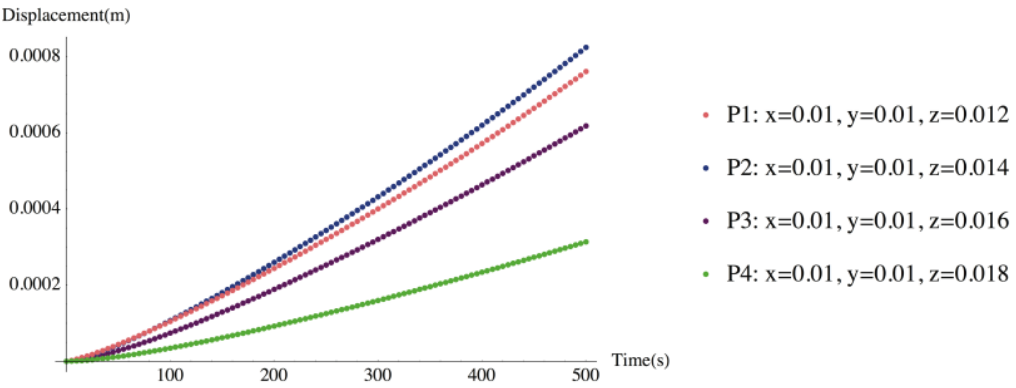

Fig. 8. Displacement changing at points $\mathrm{P} 1, \mathrm{P} 2, \mathrm{P} 3$ and $\mathrm{P} 4$.

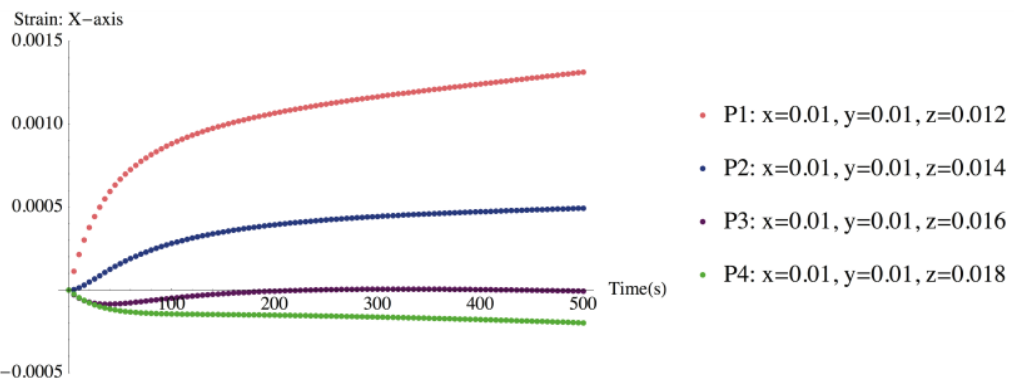

Fig. 9. Strain distribution along $\mathrm{x}$-axis at points $\mathrm{P}_{1}-\mathrm{P}_{4}$.

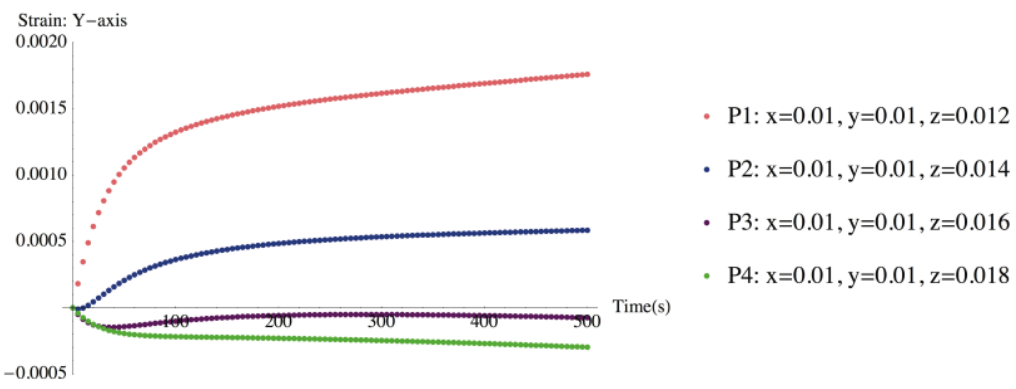

Fig. 10. Strain distribution along y-axis at points P1-P4.

Figure 7 shows a close look of the thermo-mechanical deformations with respect to temperature change, at a series of points within the deformed tumor model: point $\mathrm{P}_{1}(\mathrm{x}=0.01, \mathrm{y}=0.01, \mathrm{z}=0.012)$, 
point $\mathrm{P}_{2}(\mathrm{x}=0.01, \mathrm{y}=0.01, \mathrm{z}=0.014)$, point $\mathrm{P}_{3}(\mathrm{x}=0.01, \mathrm{y}=0.01, \mathrm{z}=0.016)$ and $\mathrm{P}_{4}(\mathrm{x}=0.01, \mathrm{y}=0.01$, $\mathrm{z}=0.018)$. By tracing the displacement of point $\mathrm{P}_{1}$ with respect to its temperature, as shown in Figure 7 , it can be seen that both its $\mathrm{x}$ and $\mathrm{y}$-axis displacements were equaled zero. Similarly, there would be no $\mathrm{x}$ and $\mathrm{y}$-axis displacement for points $\mathrm{P}_{2}-\mathrm{P}_{4}$. Thus, only the $\mathrm{z}$-axis displacement change of these four points was focused on next. Within the time range from 0 to 500 seconds, curves representing four different points respectively in Figure 8, show quite similar changing trend, moreover, the point farthest from the center of heating had smallest deformation as compared with other selected points. However, compared with other two points $\left(\mathrm{P}_{3}, \mathrm{P}_{4}\right)$ that are all further away from the heating point than $P_{1}$ is, the displacement of $P_{2}$ is surprisingly becoming larger and larger than $P_{1}$ while approaching the end of heating. To explain this, one has to find the fundamental factor that affecting the deformation of each target point, because presently, there is still no experimental data on strain and stress field available to make a verification of this simulation results. By referring to the governing equation, Eq. (7) and its discretized form Eq. (9), this factor can finally be concluded to be the derivative of $\tau$ (temperature change) with respect to the point position along z-axis, here, defined as $\mathrm{S}_{1}, \mathrm{~S}_{2}, \mathrm{~S}_{3}$ and $\mathrm{S}_{4}$ for points $\mathrm{P}_{1}-\mathrm{P}_{4}$, respectively. If we zoom in the plot, it is easy to find that at the beginning of simulation, $S_{1}$ is larger than $S_{2}$, but as the heat continually applied, $S_{2}$ become larger and larger than $S_{1}$. Comparing with other two values, $\mathrm{S}_{3}$ and $\mathrm{S}_{4}$, which are consistently smaller than $\mathrm{S}_{1}$ as their position denotes, the significantly changed value of $\mathrm{S}_{2}$ mathematically explains the surprise displacement of $\mathrm{P}_{2}$.

\subsection{Strain distribution}

To study the strain distribution within the tumor tissue as the result of temperature variation, points P1-P4 mentioned in the above section, were further analyzed based on the strain field. Figure 9, Figures 10 and 11 show the strain distribution of points P1-P4 along x, y and z-axis with respect to applied heating duration, from which it is observed that the point nearest the heating center encountered positive strain. The strain increased rapidly in the early time of heating, until after a certain point it grown gradually even the same heating was applied thereafter. Points far away from the heating center were more likely to have negative strain as compared with points near the heating sources. As an explanation of the combined zero displacement and non-zero strain at points $\mathrm{P}_{1}-\mathrm{P}_{4}$ (along the $\mathrm{x}$ and $\mathrm{y}$-axis direction, shown in Figures 8-10), one can refer to the displacements of their neighboring points. Similar as shown in Figure 6, it was easy to identify that $\mathrm{P}_{1}-\mathrm{P}_{4}$ were either of tension or compression in both directions, which explained the non-zero strain, but their displacements along $\mathrm{x}$ and $\mathrm{y}$-axis directions were all zero.

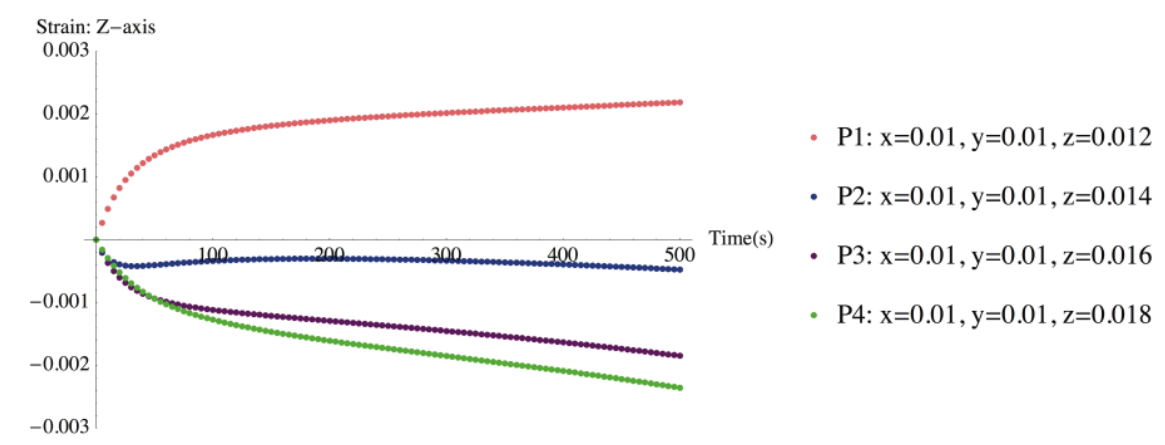

Fig. 11. Strain distribution along z-axis at points $\mathrm{P}_{1}-\mathrm{P}_{4}$ with respect to time. 
Table 2

Timing performance

\begin{tabular}{ll}
\hline Number of points & Time cost for one iteration (Second) \\
\hline 1331 & 83.3 \\
\hline 1000 & 34.7 \\
\hline 729 & 10.7 \\
\hline 512 & 2.9 \\
\hline 343 & 1.0 \\
\hline 216 & 0.4 \\
\hline
\end{tabular}

In this article, simulations were conducted on an Intel Core i7 at $3.4 \mathrm{GHz}$ PC with 8 GB RAM memory. With one external stimulus, depending on the utilized number of mesh points, the computational time for one deformation iteration is given in Table 2.

\section{Conclusion and future work}

Current thermal ablation therapy mainly relies on the quantitative temperature indication to control the delivered heat energy for cancer treatment, which is ineffective and inaccurate. This paper presents a method for prediction and analysis of thermally induced mechanical deformation for thermal ablation. This method integrates the bio-heating process and thermal induced mechanical deformation to simultaneously simulate and analyze both the heating and thermo-mechanical deformation process within the tissues. The finite difference scheme and numerical algorithms are utilized to construct the proposed thermal-mechanical soft tissue model. Experimental results demonstrate the efficiency of the proposed method. The thermal-mechanical soft tissue modeling technique established in the proposed method can be used to provide the real-time visual feedback during the process of thermal ablation for surgeons to effectively control the delivered heat energy for cancer treatment.

Future research works focusing on improvement of the proposed method include the following aspects. Firstly, the proposed method only considers regular volume meshes. It will be extended to deal with complex irregular volume meshes using the finite element scheme. Secondly, with different types of heating sources, such as contact heat, acoustic energy, and electromagnetic energy, the energy absorption rate and form are different. Therefore, the governing heat transfer equation should be modified to consider these different factors. Thirdly, the cooling effects caused by the surrounding blood vessels actually play a very import role in thermal ablation. These effects should also to be considered in the proposed model. Finally, as thermal ablation involves the movement of heating tools, particular for overlapping ablations in order to completely treat intermediate and large tumors. This makes the thermal-induced mechanical deformation always coupled with pure mechanical deformation. The current FDM model can be improved by coupling mechanical deformation caused by mechanical loads, with the thermal mechanical deformation caused by thermal loads.

\section{Acknowledgement}

This research is supported by the Australian Research Council (ARC) Discovery Grants (DP0986814 and DP110104970). 


\section{References}

[1] B. Zhang, M. Moser, E. Zhang and W.J. Zhang, Radiofrequency ablation technique in the treatment of liver tumours: Review and future issues, J. Med. Eng. Technol. 37 (2013), 150-159.

[2] G.S. Edwards, Mechanisms for soft tissue ablation and the development of alternative medical lasers based on investigations with mid-infrared free electron lasers, Laser \& Photonics Reviews 3 (2009), 545-555.

[3] P. Prakash, Theoretical modeling for hepatic microwave ablation, Open Biomed. Eng. J. 4 (2010), $27-38$.

[4] B.J. O’Daly, E. Morris, G.P. Gavin, J.M. O’Byrne and G.B. McGuinness, High-power low-frequency ultrasound: A review of tissue dissection and ablation in medicine and surgery, Journal of Materials Processing Technology 200 (2008), 38-58.

[5] C.P. Lau, Y.T. Tai and P.W. Lee, The effects of radiofrequency ablation versus medical therapy on the quality-of-life and exercise capacity in patients with accessory pathway-mediated supraventricular tachycardia: a treatment comparison study. Pacing Clin Electrophysiol, 18 (1995), pp. 424-432.

[6] P. Prakash, V.A. Salgaonkar, S.J. Scott, P. Jones, D. Hensley, A. Holbrook, J. Plata, G. Sommer and C.J. Diederich, MR guided thermal therapy of pancreatic tumors with endoluminal, intraluminal and interstitial catheter-based ultrasound devices: Preliminary theoretical and experimental investigations, SPIE BiOS 8584 (2013), 85840V-1$85840 \mathrm{~V}-10$.

[7] H. Watanabe, N. Yamazaki, Y. Kobayashi, T. Miyashita, T. Ohdaira, M. Hashizume and M.G. Fujie, Estimation of intraoperative blood flow during liver rf ablation using a finite element method-based biomechanical simulation, 2011 Annual International Conference of the Ieee Engineering in Medicine and Biology Society (Embc) (2011), 7441-7445.

[8] H. Watanabe, N. Yamazaki, Y. Isobe, X.W. Lu, Y. Kobayashi, T. Miyashita, T. Ohdaira, M. Hashizume and M.G. Fujie, Validation of accuracy of liver model with temperature-dependent thermal conductivity by comparing the simulation and in vitro RF ablation experiment, 2012 Annual International Conference of the Ieee Engineering in Medicine and Biology Society (Embc) (2012), 5712-5717.

[9] P. Rattanadecho and P. Keangin, Numerical study of heat transfer and blood flow in two-layered porous liver tissue during microwave ablation process using single and double slot antenna, International Journal of Heat and Mass Transfer 58 (2013), 457-470.

[10] F. Xu and T. Lu, Introduction to skin biothermomechanics and thermal pain: Springer (2011), 42-57.

[11] B. Zhou, F. Xu, C.Q. Chen and T.J. Lu, Strain rate sensitivity of skin tissue under thermomechanical loading, Philosophical Transactions of the Royal Society a-Mathematical Physical and Engineering Sciences 368 (2010), 679690.

[12] W.S. Shen and J. Zhang, Modeling and numerical simulation of bioheat transfer and biomechanics in soft tissue, Mathematical and Computer Modelling 41 (2005), 1251-1265.

[13] W.S. Shen, J. Zhang and F.Q. Yang, Skin thermal injury prediction with strain energy, International Journal of Nonlinear Sciences and Numerical Simulation 6 (2005), 2317-2328.

[14] J. Jimenez-Lozano, P. Vacas-Jacques and W. Franco, Thermo-elastic response of cutaneous and subcutaneous tissues to noninvasive radiofrequency heating, Proceedings of the 2012 COMSOL Conference in Boston (2012).

[15] I. Chang, Finite element analysis of hepatic radiofrequency ablation probes using temperature-dependent electrical conductivity, Biomedical Engineering Online 2 (2003), 1-18.

[16] Y. Zhang, D.B. Goldgof, S. Sarkar and L.V. Tsap, A modeling approach for burn scar assessment using natural features and elastic property, IEEE Transactions on Medical Imaging 23 (2004), 1325-1329.

[17] H. Watanabe, Y. Kobayashi, M. Hashizume and M.G. Fujie, Modeling the temperature dependence of thermophysical properties: Study on the effect of temperature dependence for RFA, 31st Annual International Conference of the IEEE Engineering in Medicine and Biology Society: Engineering the Future of Biomedicine, EMBC 2009, Minneapolis, MN, United states (2009), 5100-5105.

[18] D.W. Evans, E.C. Moran, P.M. Baptista, S. Soker and J.L. Sparks, Scale-dependent mechanical properties of native and decellularized liver tissue, Biomechanics and Modeling in Mechanobiology 12 (2013), 569-580. 